\title{
Radiation and MHD effects on an oscillatory flow of a Jeffrey fluid in a circular tube
}

\author{
Dr.Bhusireddy Swaroopa, Prof. K.Ramakrishna Prasad
}

\begin{abstract}
In this paper, we studied the effects of heat transfer and MHD on oscillatory flow of Jeffrey fluid in a circular tube with radiation. The expressions for the velocity field and temperature field are obtained analytically. The effects of various emerging parameters on the velocity field and temperature field studied in detail with the help of graphs.
\end{abstract}

Index Terms - Jeffrey fluid, Reynolds number, Prandtl number, Grashof number, Nusselt number.

\section{INTRODUCTION}

The effect of heat transfer on unsteady MHD oscillatory flow of fluid in a tube is encountered in a wide range of engineering and industrial applications such as molten iron flow, recovery extraction of crude oil, geothermal systems. Many chemical engineering processes like metallurgical and polymer extrusion processes involve cooling of a molten liquid being stretched in a cooling system. Some polymers fluids like polyethylene oxide and polysobutylene solutions in a cetane, having better electromagnetic properties are normally used as cooling liquid as their flow can be regulated by external magnetic fields in order to improve the quality of the final product. Also, the radiative heat transfers is an important factor of thermodynamics of very high temperature systems such as electric furnaces, solar collectors, storage of nuclear wastes packed bed catalytic reactors, satellites, steel rolling, cryogenic engineering etc. The study of such flow under the influence of magnetic field and heat transfer has attracted the interest of many investigators and researchers. Unsteady and oscillatory flow of viscous fluids in locally constricted, rigid, axisymmetric tubes at low Reynolds number has been studied by Ramachandra Rao and Devanathan (1973), Hall(1974), Chaturani and Upadyaya (1978) and Schneck and Ostrach (1975). Haldar (1987) have considered the oscillatory flow of a blood through an artery with a mild constriction. Ogulu and Abbey (2005) have studied the effect of heat transfer on the motion of blood in a diseased artery has been modeled under the optically thin fluid assumption. Several other workers, Misra and Singh (1987), Ogulu and Alabraba (1992), Tay and Ogulu (1997) and Elshahed (2003), to mention but a few, have in one way or the other modeled and studied the flow of blood through a rigid tube under the influence of pulsatile pressure gradient. Arunakumari et al. (2012) investigated the influence of heat transfer on MHD oscillatory flow of Jeffery fluid in a Channel. Unsteady Flow of a Jeffrey Fluid in an

Dr. Bhusireddy Swaroopa, Asst. Prof. in Mathematics, Governmen Degree College For Women, Srikalahasti, Chittoor (Dist.), Andhra Pradesh.-517644 Mobile No. 9959157622

Prof. K. Ramakrishna Prasad,(Rtd.) Principal \& Professor of Mathematics, S. V. University, Tirupati, Chittoor(Dist.), Andhra pradesh 517502 elastic tube with stenosis was investigated by Sreenadh et al (2012).

In view of these, we studied the effects of heat transfer and MHD on oscillatory flow of Jeffrey fluid in a circular tube with radiation. The expressions for the velocity field and temperature field are obtained analytically. The effects of various emerging parameters on the velocity field and temperature field studied in detail with the help of graphs.

\section{II. .MATHEMATICAL FORMULATION}

We consider an oscillatory flow of a Jeffrey fluid in a heated uniform cylindrical tube of constant radius $R$ in the presence of uniform magnetic field $B_{0}$. The wall of the tube is maintained at a temperature $T_{w}$. We choose the cylindrical coordinates $(r, \theta, z)$ such that $r=0$ is the axis of symmetry. The flow is considered as axially symmetric and fully developed. The geometry of the flow is shown in Fig. 1.

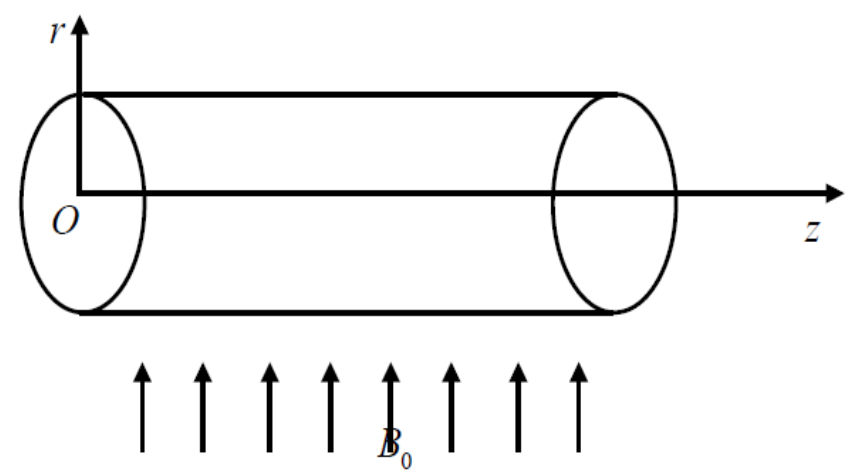

Fig. 1 The physical model of the

Fig. 1 The physical model of the problem

The equations governing the flow (Kavitha and Ramakrishna Prasad, 2012) are given by

$$
\begin{gathered}
\rho \frac{\partial w}{\partial t}=-\frac{\partial p}{\partial z}+\frac{\mu}{1+\lambda_{1}}\left(\frac{\partial^{2} w}{\partial r^{2}}+\frac{1}{r} \frac{\partial w}{\partial r}\right)-\sigma B_{0}^{2} w+\rho g \beta\left(T-T_{\infty}\right) \\
\rho c_{p} \frac{\partial T}{\partial t}=k_{0}\left(\frac{\partial^{2} T}{\partial r^{2}}+\frac{1}{r} \frac{\partial T}{\partial r}\right)-\frac{\partial q}{\partial r}(2)
\end{gathered}
$$

where $\rho$ is the fluid density, $\lambda_{1}$ is the ratio of relaxation to retardation times, $\mu$ is the fluid viscosity, $p$ is the pressure, $w$ is the velocity component in $z$ - direction, $g$ is the acceleration due to gravity, $\sigma$ is electrical oconductivity, $\beta$ 
coefficient of thermal expansion, $T$ is the temperature, $k_{0}$ is the thermal conductivity and $c_{p}$ is the specific heat at constant pressure. Following Cogley et al. (1968), it is assumed that the fluid is optically thin with a relatively low density and the radiative heat flux is given by

$$
\frac{\partial q}{\partial y}=4 \alpha^{2}\left(T_{\infty}-T\right)
$$

here $\alpha$ is the mean radiation absorption coefficient.

The appropriate boundary conditions are

$$
\begin{gathered}
w=0, T=T_{w} \quad \text { at } \quad r=R \\
\frac{\partial w}{\partial r}=0, T=T_{\infty} \quad \text { at } \quad r=0
\end{gathered}
$$

Introducing the following non-dimensional variables

$$
\begin{aligned}
& \bar{r}=\frac{r}{R}, \bar{z}=\frac{z}{R}, \bar{t}=\frac{w_{0} t}{R}, \quad \alpha^{2}=\frac{\rho R^{2}}{\mu} \\
& \bar{w}=\frac{w}{w_{0}}, \\
& \bar{p}=\frac{p-p_{w}}{\mu}, \quad \theta=\frac{T-T_{\infty}}{T_{w}-T_{\infty}} \\
& \operatorname{Pr}=\frac{\mu c_{p}}{k_{0}}, \quad \operatorname{Re}=\frac{\rho w_{0} R}{\mu},
\end{aligned}
$$

into the Eqs. (1) and (2), we get (after dropping bars) $\operatorname{Re} \frac{\partial w}{\partial t}=-\lambda \frac{d p}{d z}+\frac{1}{1+\lambda_{1}}\left[\frac{\partial^{2} w}{\partial r^{2}}+\frac{1}{r} \frac{\partial w}{\partial r}\right]-M^{2} w+\frac{G r}{\operatorname{Re}} \theta$

$$
\operatorname{Pr} \operatorname{Re} \frac{\partial \theta}{\partial t}=\frac{\partial^{2} \theta}{\partial r^{2}}+\frac{1}{r} \frac{\partial \theta}{\partial r}+N^{2} \theta
$$

where $\operatorname{Pr}$ is the Prandtl number, $M=R B_{0} \sqrt{\frac{\sigma}{\mu}}$ is the

Darcy number and $\mathrm{Re}$ is the Reynolds number.

The corresponding non-dimensional boundary conditions are

$$
\begin{aligned}
& w=0, \quad \theta=1 \quad \text { at } \quad r=1 \\
& \frac{\partial w}{\partial r}=0, \quad \frac{\partial \theta}{\partial r}=0 \quad \text { at } \quad r=0
\end{aligned}
$$

\section{SOLUTION}

It is fairly unanimous that, the pumping action of the heart results in a pulsatile blood flow so that we ca represent the pressure gradient (pressure in the left ventricle) as

$-\frac{d p}{d z}=p_{0} e^{i w t}$

and flow variables expresses as

$$
\begin{aligned}
& \theta(y, t)=\theta_{0}(r) e^{i w t} \\
& w(y, t)=w_{0}(r) e^{i w t}
\end{aligned}
$$

Substituting Eqs. (8) - (10) into Eqs. (5) and (6) and solving the resultant equations subject to the boundary conditions in (7), we obtain

$$
\theta_{0}=\frac{I_{0}(\Omega r)}{I_{0}(\Omega)}
$$

$w_{0}=\frac{G r}{\operatorname{Re}} \frac{\left(1+\lambda_{1}\right)}{\left(\beta_{1}^{2}+\Omega^{2}\right)}\left[\frac{I_{0}\left(\beta_{1} r\right)}{I_{0}\left(\beta_{1}\right)}-\frac{I_{0}(\Omega r)}{I_{0}(\Omega)}\right]+\frac{\lambda p_{0}}{\beta_{1}^{2}}\left(1+\lambda_{1}\right)\left[1-\frac{I_{0}\left(\beta_{1} r\right)}{I_{0}\left(\beta_{1}\right)}\right]$

Here $\Omega^{2}=i \omega \operatorname{Pr} \operatorname{Re}-N^{2}$

$\beta_{1}^{2}=\left(M^{2}+i \omega \operatorname{Re}\right)\left(1+\lambda_{1}\right)$ and $I_{0}(x)$ is the modified Bessel function of first kind of order zero.

Thus the temperature distribution and the axial velocity are given by

$\theta=\frac{I_{0}(\Omega r)}{I_{0}(\Omega)} e^{i \omega t}$

$w=\left(\frac{G r}{\operatorname{Re}} \frac{\left(1+\lambda_{1}\right)}{\left(\beta_{1}^{2}+\Omega^{2}\right)}\left[\frac{I_{0}\left(\beta_{1} r\right)}{I_{0}\left(\beta_{1}\right)}-\frac{I_{0}(\Omega r)}{I_{0}(\Omega)}\right]+\frac{\lambda p_{0}}{\beta_{1}^{2}}\left(1+\lambda_{1}\right)\left[1-\frac{I_{0}\left(\beta_{1} r\right)}{I_{0}\left(\beta_{1}\right)}\right]\right) e^{i \omega t}$ given by

$\tau=\left.\frac{\partial u}{\partial r}\right|_{r=1}=\left(\frac{G r}{\operatorname{Re}} \frac{\left(1+\lambda_{1}\right)}{\left(\beta_{1}^{2}+\Omega^{2}\right)}\left[\frac{\beta_{1} I_{1}\left(\beta_{1}\right)}{I_{0}\left(\beta_{1}\right)}-\frac{\Omega I_{1}(\Omega)}{I_{0}(\Omega)}\right]+\frac{\lambda p_{0}}{\beta_{1}^{2}}\left(1+\lambda_{1}\right)\left[1-\frac{\beta_{1} I_{1}\left(\beta_{1}\right)}{I_{0}\left(\beta_{1}\right)}\right]\right) e^{i \omega t}$

The rate of heat transfer coefficient in terms of Nusselt number on the boundary of the tube is

$$
N u=\left.\frac{\partial \theta}{\partial r}\right|_{r=1}=\frac{\Omega I_{1}(\Omega)}{I_{0}(\Omega)} e^{i \omega t}
$$

\section{DISCUSSION OF THE RESULTS}

Fig. 2 shows the effects of material parameter $\lambda_{1}$ on $w$ for $M=1, \quad N=1, \quad p=1$, $\omega=10, \lambda=0.5, \operatorname{Pr}=0.7, G r=1, \operatorname{Re}=1$ and $t=0.1$. It is observed that, the axial velocity $w$ increases at the axis of tube with increasing material parameter $\lambda_{1}$, while it decreases near the tube wall with increasing $\lambda_{1}$.

Effects of Hartmann number $M$ on $w$ for $\lambda_{1}=0.3, \quad N=1, \quad p=1, \quad \omega=10, \quad \lambda=0.5$, $\operatorname{Pr}=0.7, G r=1, \operatorname{Re}=1$ and $t=0.1$ is shown in Fig. 3. It is found that the axial velocity $w$ decreases with increasing Hartmann number $M$.

Fig. 4 depicts the effects of Prandtl number $\mathrm{Pr}$ on $w \quad$ for $\quad \lambda_{1}=0.3$ $N=1, \quad p=1 \quad, \quad \omega=10 \quad, \quad M=1$, $\lambda=0.5, G r=1, \operatorname{Re}=1$ and $t=0.1$. It is noted that, the axial velocity $w$ increases on increasing Prandtl number $\operatorname{Pr}$. 
Effects of radiation parameter $N$ on $w$ for $\lambda_{1}=0.3, p=1, \omega=10, G r=1, \quad \lambda=0.5$, $M=1, \operatorname{Pr}=0.7, \operatorname{Re}=1$ and $t=0.1$ is presented in Fig. 5. It is observed that, the axial velocity $w$ increases with increasing $N$.

Fig. 6 depicts the effects of Grashof number $G r$ on $w$ for $\lambda_{1}=0.3, N=1, p=1, \omega=10, M=1$, $\lambda=0.5, \operatorname{Pr}=0.7, \operatorname{Re}=1$ and $t=0.1$. It is noted that, the axial velocity $w$ increases at the axis of tube on increasing Grashof number $G r$, while it decreases near the tube wall with increasing $G r$.

The effect of Reynolds number $\operatorname{Re}$ on $w$ for $N=1, \lambda_{1}=0.3, p=1, \omega=10, \lambda=0.5, M=1$, $\operatorname{Pr}=0.7, \operatorname{Re}=1$ and $t=0.1$ is shown in Fig. 7. It is found that, the axial velocity $w$ decreases with an increase in Reynolds number $\mathrm{Re}$.

Fig. 8 shows the effect of $\lambda$ on $w$ for $\lambda_{1}=0.3$, $N=1, M=1, P r=0.7, G r=1, p=1, \omega=10$, $\mathrm{Re}=1$ and $t=0.1$. It is observed that, the axial velocity $w$ increases on increasing $\lambda$.

Effect of oscillating parameter $\omega$ on $w$ for $M=1, p_{0}=1, \lambda_{1}=0.5, \lambda=0.5, \quad \operatorname{Pr}=0.7$, $G r=1, N=1, \operatorname{Re}=1$ and $t=0.1$ is depicted in Fig. 9. It is found that, the axial velocity $w$ oscillates with $\omega$.

Fig. 10 shows the effect of Prandtl number $\mathrm{Pr}$ on $\theta$ for $\omega=10, N=1, \operatorname{Re}=1$ and $t=0.1$. It is found that, the temperature $\theta$ decreases with increasing Prandtl number $\mathrm{Pr}$

Effect of radiation parameter $N$ on $\theta$ for $\omega=10, \operatorname{Pr}=0.7, \operatorname{Re}=1$ and $t=0.1$ is shown in Fig. 11. It is noted that, the temperature $\theta$ increases with increasing $N$.

Fig. 12 depicts the effect of Reynolds number $\mathrm{Re}$ on $\theta$ for $\omega=10, \operatorname{Pr}=0.7, N=1$ and $t=0.1$. It is observed that, the temperature $\theta$ decreases with increasing Reynolds number $\mathrm{Re}$.

Effects of oscillating parameter $\omega$ on $\theta$ for $\operatorname{Pr}=0.7, N=1, \operatorname{Re}=1$ and $t=0.1$ is depicted in Fig. 13. It is found that, the temperature $\theta$ increases with increasing $\omega$.

Table-1 shows the effects of various physical parameters like $\lambda_{1}, G r, \operatorname{Re}, \lambda, M, \operatorname{Pr}$ and $N$ on the skin friction $\tau$ at the tube wall $r=1$. It is found that, the skin friction increases for increasing the parameters $\lambda_{1}$, $G r, \operatorname{Re}$ and $\lambda$, while it decreases $M, \operatorname{Pr}$ and $N . r=1$. It is observed that, the Nusselt number increases with increasing $\operatorname{Pr}$ and $\mathrm{Re}$, while it decreases with increasing $N$.

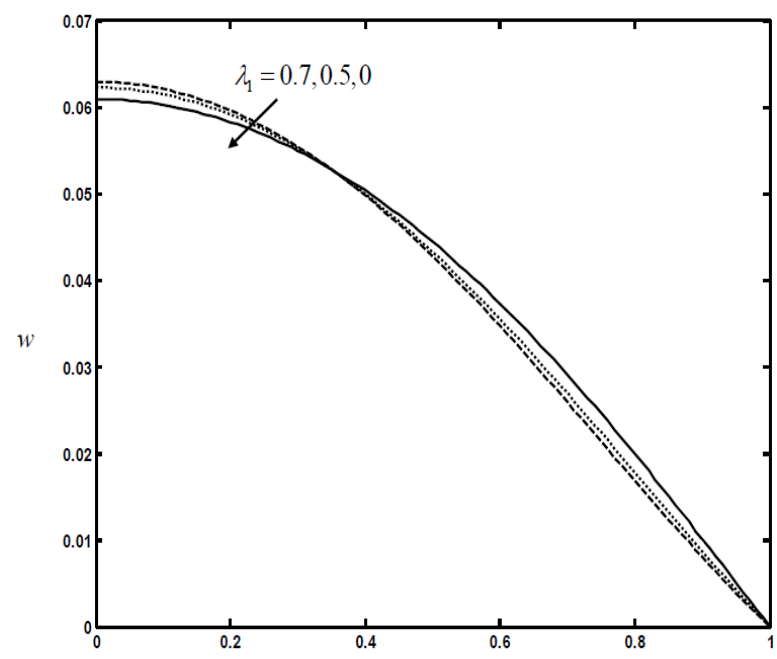

Fig. 2. Effects of material parameter $\lambda_{1}$ on $w$ for $M=1$, $p_{0}=1, \omega=10, \lambda=0.5, \operatorname{Pr}=0.7, G r=1$, $N=1, \operatorname{Re}=1$ and $t=0.1$.

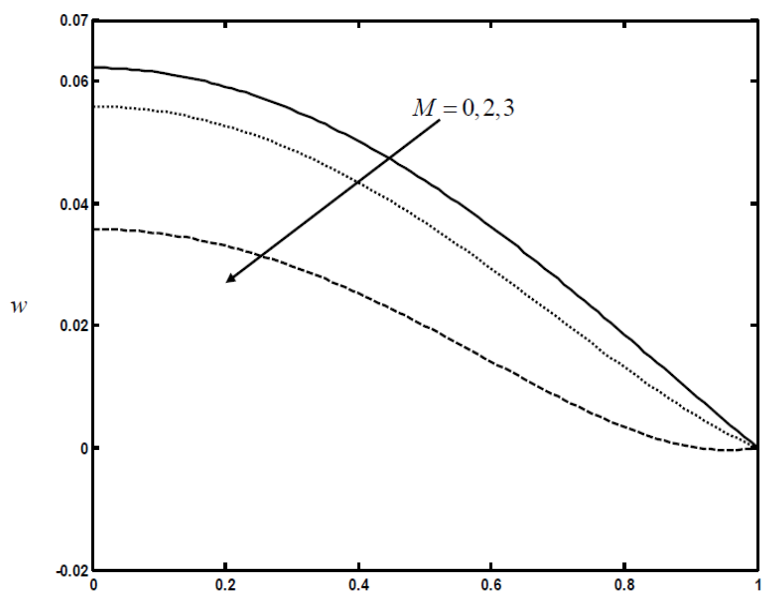

Fig. 3. Effects of Hartmann number $M$ on $w$ for $\lambda_{1}=0.5, p_{0}=1, \omega=10, \lambda=0.5$, $\operatorname{Pr}=0.7, G r=1, N=1, \operatorname{Re}=1$ and $t=0.1$.

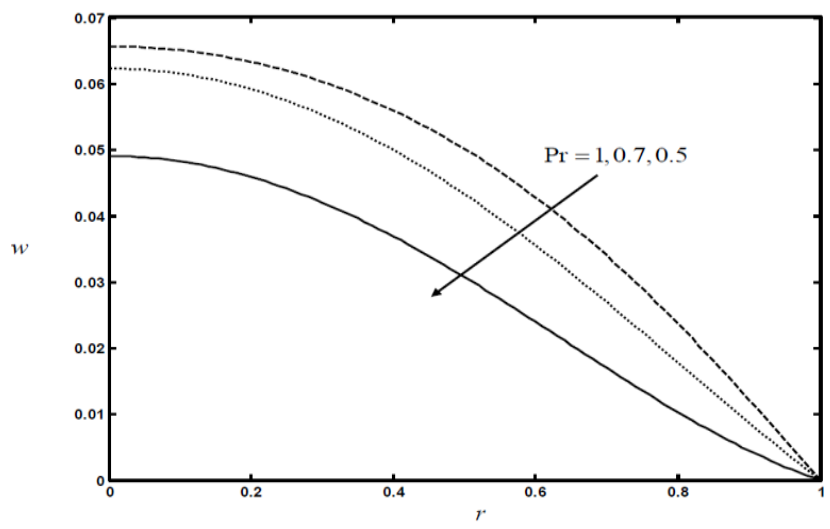


Fig. 4. Effects of Prandtl number $\lambda_{1}$ on $w$ for $M=1$,

$p_{0}=1, \omega=10, \lambda=0.5, \lambda_{1}=0.5, G r=1$, $N=1, \operatorname{Re}=1$ and $t=0.1$.

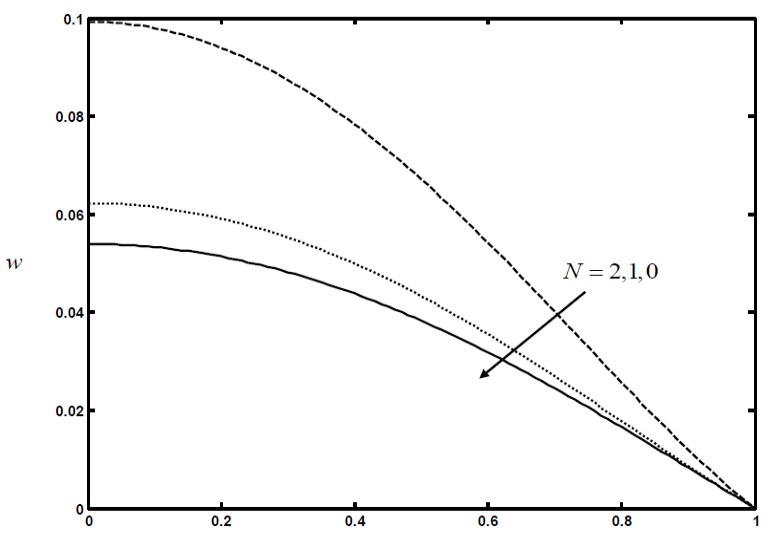

Fig. 5. Effects of radiation parameter $N$ on $w$ for $M=1, p_{0}=1, \omega=10, \lambda=0.5$, $\operatorname{Pr}=0.7, G r=1, \lambda_{1}=0.5, \operatorname{Re}=1$ and $t=0.1$.

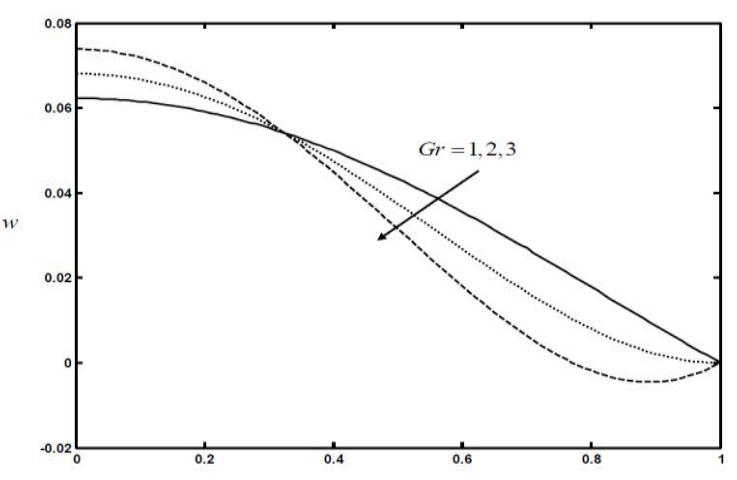

Fig. 6. Effects of Grashof number $G r$ on $w$ for $M=1$, $p_{0}=1, \omega=10, \lambda=0.5, \operatorname{Pr}=0.7, \lambda_{1}=0.5$, $N=1, \operatorname{Re}=1$ and $t=0.1$.

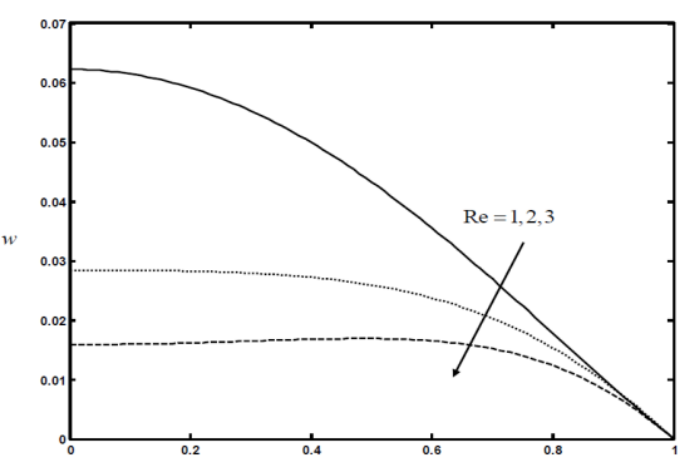

Fig. 7. Effects of Reynolds number $\operatorname{Re}$ on $w$ for $M=1$, $p_{0}=1, \omega=10, \lambda=0.5, \operatorname{Pr}=0.7, G r=1$, $N=1, \lambda_{1}=0.5$ and $t=0.1$.

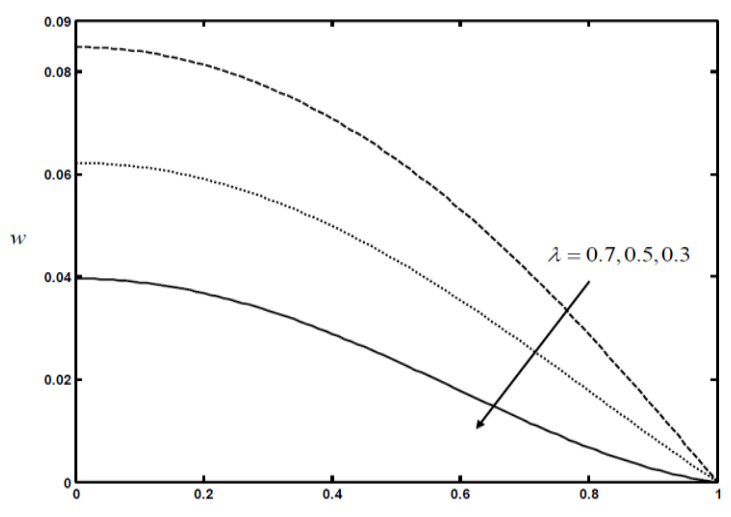

Fig. 8. Effects of pressure constant $\lambda$ on $w$ for $M=1$, $p_{0}=1, \omega=10, \lambda_{1}=0.5, \operatorname{Pr}=0.7, G r=1$, $N=1, \operatorname{Re}=1$ and $t=0.1$.

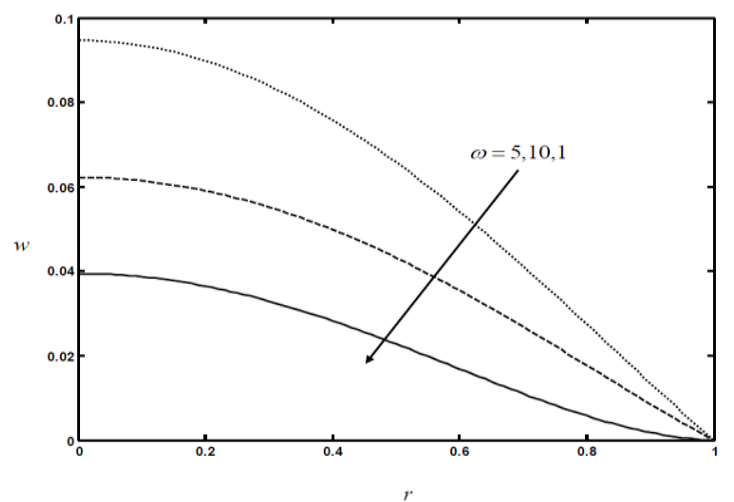

Fig. 9. Effects of oscillating parameter $\omega$ on $w$ for $M=1, p_{0}=1, \lambda_{1}=0.5, \lambda=0.5$, $\operatorname{Pr}=0.7, G r=1, N=1, \operatorname{Re}=1$ and $t=0.1$

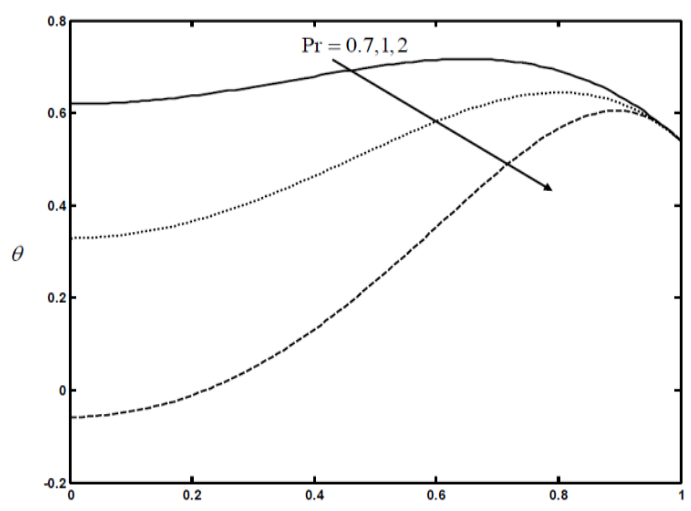

Fig. 10. Effects of Prandtl number $\operatorname{Pr}$ on $\theta$ for $\omega=10$, $N=1, \operatorname{Re}=1$ and $t=0.1$. 


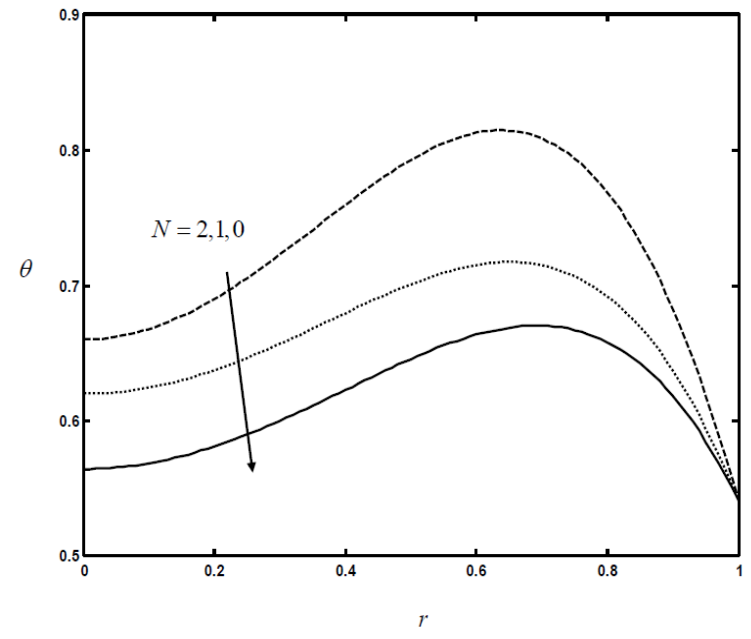

Fig. 11. Effects of radiation parameter $N$ on $\theta$ for $\omega=10, \operatorname{Pr}=0.7, \operatorname{Re}=1$ and $t=0.1$.

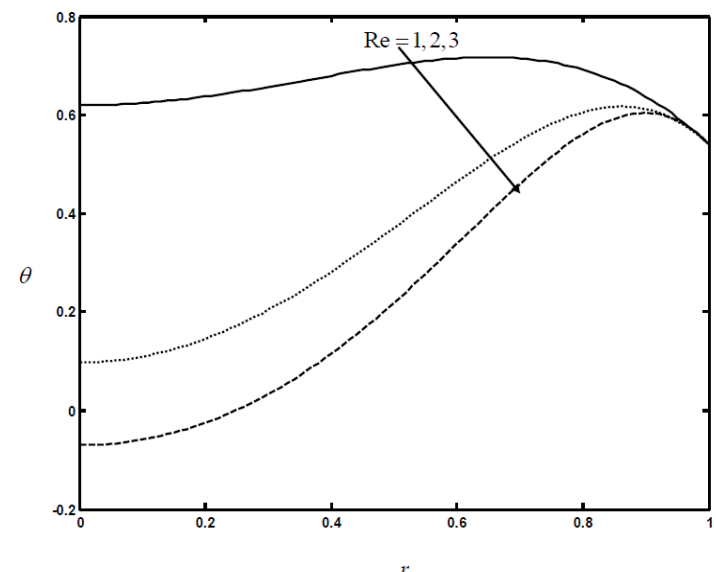

Fig. 12. Effects of Reynolds number $\operatorname{Re}$ on $\theta$ for $\omega=10, \operatorname{Pr}=0.7, N=1$ and $t=0.1$.

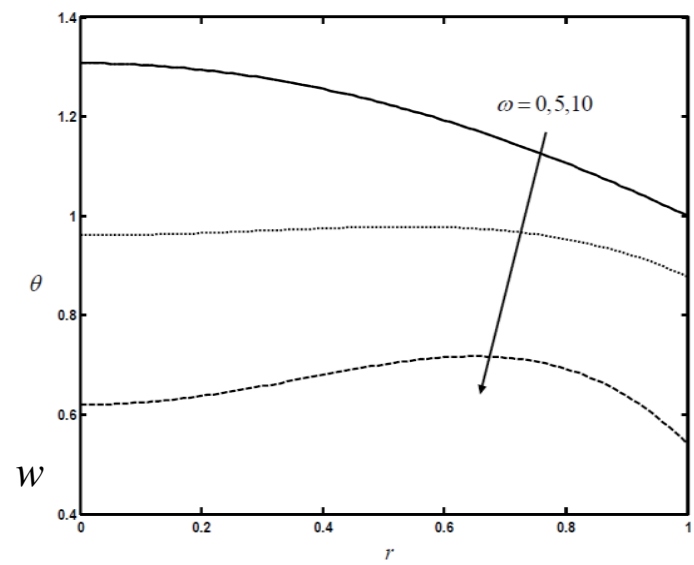

Fig. 13. Effects of oscillating parameter $\omega$ on $\theta$ for $\operatorname{Pr}=0.7, N=1, \operatorname{Re}=1$ and $t=0.1$.

Table - 1: The Skin friction $\tau$ at the tube wall $r=1$

\begin{tabular}{|l|l|l|l|l|l|l|l|}
\hline$\lambda_{1}$ & $M$ & $\operatorname{Pr}$ & $N$ & $G r$ & $\operatorname{Re}$ & $\lambda$ & $\tau$ \\
\hline 0.5 & 1 & 0.7 & 1 & 1 & 1 & 0.5 & -0.1118 \\
& & & & & & & \\
\hline 0.7 & 1 & 0.7 & 1 & 1 & 1 & 0.5 & -0.1072 \\
\hline 0.5 & 2 & 0.7 & 1 & 1 & 1 & 0.5 & -0.1189 \\
\hline 0.5 & 1 & 1 & 1 & 1 & 1 & 0.5 & -0.1308 \\
\hline 0.5 & 1 & 0.7 & 2 & 1 & 1 & 0.5 & -0.1609 \\
\hline 0.5 & 1 & 0.7 & 1 & 2 & 1 & 0.5 & -0.0809 \\
\hline 0.5 & 1 & 0.7 & 1 & 1 & 2 & 0.5 & -0.0814 \\
\hline 0.5 & 1 & 0.7 & 1 & 1 & 1 & 0.7 & -0.1072 \\
\hline
\end{tabular}

Table - 2: The Nusselt number $N u$ at the tube wall $r=1$

\begin{tabular}{|l|l|l|l|}
\hline $\operatorname{Pr}$ & $N$ & $\operatorname{Re}$ & $N u$ \\
\hline 0.7 & 1 & 1 & 1.2113 \\
\hline 1 & 1 & 1 & 1.6620 \\
\hline 0.7 & 2 & 1 & 1.1200 \\
\hline 0.7 & 1 & 2 & 2.0789 \\
\hline
\end{tabular}

\section{CONCLUSIONS}

In view of these, we studied the effects of heat transfer and MHD on oscillatory flow of Jeffrey fluid in a circular tube with radiation. The expressions for the velocity field and temperature field are obtained analytically. It is found that the velocity $w$ increases with increasing $\lambda_{1}, \operatorname{Pr}, N, G r, \omega$ and $\lambda$, while it decreases with increasing $M$ and $\operatorname{Re}$; the temperature increases with increasing $\operatorname{Pr}, N$ and $\omega$, while it decreases with increasing $\mathrm{Re}$; the skin friction increases for increasing the parameters $\lambda_{1}, G r, \operatorname{Re}$ and $\lambda$, while it decreases $M, \operatorname{Pr}$ and $N$ and the Nusselt number increases with increasing $\operatorname{Pr}$ and $\operatorname{Re}$, while it decreases with increasing $N$.

\section{REFERENCES}

[1] B. Aruna Kumari, K. Ramakrishna Prasad and K. Kavita, Slip Effects on MHD Oscillatory Flow of Jeffrey Fluid in a Channel with Heat Transfer. Int. J. Math. Arch. 3 (8) (2012), 2903 - 2911.

[2] P. Chaturani and V. S. Upadhya, Pulsatile flow of a couple stress fluid through circular tubes with application to blood flow, Biorheology 15 (1978), 193-201.

[3] A. C. Cogley, S. E. Gilles, W. G. Vincenti, S. Ishimoto, Differential approximation for radiative heat transfer in a non grey gas near equilibrium. Am. Inst. Aeron. Astron. 6(3) (1968), 551-553.

[4] Elshahed, Pulsatile flow of blood through a stenosed porous medium under periodic body acceleration, Appl. Mathematics and Computation, 138(2003), 479-488.

[5] K. Haldar, Oscillatory flow of blood in a stenosed artery, Bulletin of Mathematical Biology, 49(1987), 279-287.

[6] P. Hall, Unsteady viscous flow in a pipe of slowly varying cross section, J. Fluid Mech. 64(1974), 209-226.

[7] K. Kavitha and K. Ramakrishna Prasad, Effects of MHD and heat transfer on an oscillatory flow of Jeffrey fluid in a tube. International Journal of Dynamics of Fluids, 8(1) (2012), 1-10.

[8] J. C. Misra and S. I. Singh, Bulletin of Mathematical Biology, 49(1987), 257.

[9] A. Ogulu and M. A. Alabraba, Acta Physica Hungarica, 72(1992), 223. 
[10] Ogulu and T. M. Abbey, Simulation of heat transfer on an oscillatory blood flow in an indented porous artery, International Communications in Heat and Mass Transfer, 32 (2005), 983-989

[11] A. Ramachandra Rao and R. Devanathan, Pulsatile flow in tubes of varying cross-sections, ZAMP, 24(1973), 203-213.

[12] D. J. Schneck and S. Ostrach, Pulsatile blood flow in a channel of small exponential divergence-I: The linear approximation for low Reynolds number, J. Fluids Engng. 97(1975), 353-360.

[13] S. Sreenadh, P. Devaki and R. Diwakar, Unsteady flow of Jeffrey fluid in an elastic tube with stenosis, International Conference of Fluid Dynamics and Thermodynamics Technologies, IPCSIT Vol 33 (2012), IACSIT Press, Singapore

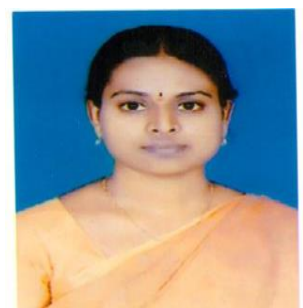

Dr. B . Swaroopa,M.Sc.,M.Phil.,Ph.D.,NET Qualified Publications:

$>$ Slip effects on the flow of a Carreau fluid through a porous medium in a planar channel under the effect of a magnetic field with peristalsis

International Journal of Engineering and Technical Research (IJETR) ISSN: 2321-0869, Volume-3, Issue-4, April 2015

$>$ Effect of Heat Transfer on Oscillatory Flow of a Fluid through a Porous Medium in a Channel with an Inclined Magnetic Field

International Journal of Computer \& Mathematical Sciences IJCMS ISSN 2347 - 8527 Volume 4, Issue 10 October 2015

$>$ Influence of radiation on MHD free convective flow of a Williamson fluid in a International Journal of Engineering and Technical Research (IJETR)vertical channel ISSN: 2321-0869 (O) 2454-4698 (P), Volume-5, Issue-2, June 2016

\section{Membership in Mathematics Bodies:}

$>$ Life Member, The Indian Society For Technical Education.

$>$ Life Member, Andhra Pradesh Society For Mathematical Sciences.

$>$ Life Member, Indian Mathematical Society.

Achievements :

$>$ Stood College First in B.Sc

$>$ Secured $2^{\text {nd }}$ rank both in S.V.U. and S.K.U. CET

$>$ Qualified ARPIT Exam, 2019 - Refresher Course in Calculus One of our student,secured State level highest marks in Three year Degree B.Sc

Qualified ARPIT Exam, 2019 - ONLINE Refresher Course in Calculus 